\title{
Heat shock factor 1 mediates the longevity conferred by inhibition of TOR and insulin/IGF-1 signaling pathways in C. elegans
}

Keunhee Seo, ${ }^{1}$ Eunseok Choi, ${ }^{1}$ Dongyeop Lee, ${ }^{1}$ Dae-Eun Jeong, ${ }^{1}$ Sung Key Jang ${ }^{1}$ and Seung-Jae Lee ${ }^{1,2}$

${ }^{1}$ Department of Life Sciences,

${ }^{2}$ School of Interdisciplinary Bioscience and Bioengineering, Pohang University of Science and Technology, Pohang, Gyeongbuk 790-784, South Korea

\section{Summary}

Target of rapamycin (TOR) signaling is an evolutionarily wellconserved pathway that regulates various physiologic processes, including aging and metabolism. One of the key downstream components of TOR signaling is ribosomal protein $\mathrm{S} 6$ kinase (S6K) whose inhibition extends the lifespan of yeast, Caenorhabditis elegans, Drosophila, and mice. Here, we demonstrate that the activation of heat shock factor 1 (HSF-1), a crucial longevity transcription factor known to act downstream of the insulin/IGF1 signaling (IIS) pathway, mediates the prolonged lifespan conferred by mutations in C. elegans S6K (rsks-1). We found that $h s f-1$ is required for the longevity caused by down-regulation of components in TOR signaling pathways, including TOR and S6K. The induction of a small heat-shock protein hsp-16, a transcriptional target of HSF-1, mediates the long lifespan of rsks-1 mutants. Moreover, we show that synergistic activation of HSF-1 is required for the further enhanced longevity caused by simultaneous down-regulation of TOR and IIS pathways. Our findings suggest that HSF-1 acts as an essential longevity factor that intersects both IIS and TOR signaling pathways.

Key words: aging; Caenorhabditis elegans; HSF-1; insulin/ IGF-1 signaling; longevity; mRNA translation; S6 kinase; target of rapamycin.

\section{Introduction}

For the last two decades, numerous genes and pathways that influence aging have been discovered using model organisms including yeast, Caenorhabditis elegans, and Drosophila melanogaster. Many of these genes and pathways have been shown to regulate lifespan in mammals as well (Fontana et al., 2010; Kenyon, 2010). Among them, insulin/IGF-1 signaling (IIS) and target of rapamycin (TOR) pathways are two of the most evolutionarily well-conserved aging-regulatory pathways (Stanfel

\section{Correspondence}

Sung Key Jang, Department of Life Sciences, Pohang University of Science and Technology, Pohang, Gyeongbuk, 790-784, South Korea. Tel..+82-54-279-2258; fax: +82-54-279-8009; e-mail: sungkey@postech.ac.kr and Seung-Jae Lee, Department of Life Sciences, Pohang University of Science and Technology, Pohang, Gyeongbuk, 790-784, South Korea. Tel.: +82-54-279-2351;

fax: +82-54-279-2199; e-mail: seungjaelee@postech.ac.kr

Accepted for publication 08 July 2013 et al., 2009; Fontana et al., 2010; Kapahi et al., 2010; Kenyon, 2010; Evans et al., 2011). Insulin/IGF-1 signaling and TOR signaling pathways link metabolic status and aging and interact with each other, although the factor(s) mediating the cross talk between these two pathways is incompletely understood.

Target of rapamycin is a protein kinase that acts as a cellular nutrient sensor to regulate various physiologic processes, including metabolism, growth, and aging (Stanfel et al., 2009; Kapahi et al., 2010; Evans et al., 2011; Laplante \& Sabatini, 2012). The TOR kinase forms two distinct complexes, TORC 1 and TORC2, which have complex-specific adaptors such as raptor and rictor, respectively (Dazert \& Hall, 2011; Laplante \& Sabatini, 2012). The activity of TORC1 is regulated by upstream signaling proteins such as Rag GTPases in response to changes in the cellular environmental conditions (Kim et al., 2008). When the nutrient supply is sufficient, TORC 1 positively regulates cellular growth and proliferation by promoting protein synthesis and/or inhibiting autophagy. To exert its effects on protein synthesis, TORC1 phosphorylates its target proteins, including ribosomal protein S6 kinase (S6K) and eukaryotic translation initiation factor 4E-binding protein 1 (4E-BP1) (Laplante \& Sabatini, 2012). S6K mediates the regulation of mRNA translation via phosphorylation of its substrates, including ribosomal protein subunit 6 (Erikson \& Maller, 1985; Laplante \& Sabatini, 2012). Recent studies have shown that genetic inhibition of TOR, S6K, or ribosomal protein subunits extends lifespan in many organisms (Fabrizio et al., 2001; Jia et al., 2004; Kapahi et al., 2004; Kaeberlein et al., 2005; Chen et al., 2007; Curran \& Ruvkun, 2007; Hansen et al., 2007; Pan et al., 2007; Syntichaki et al., 2007; Selman et al., 2009) and that treatment with the TORC 1 inhibitor rapamycin increases the lifespan of yeast, C. elegans, Drosophila, and mice (Powers et al., 2006; Harrison et al., 2009; Bjedov et al., 2010; Robida-Stubbs et al., 2012). These studies establish the TOR signaling pathway as an evolutionarily conserved agingregulatory pathway and a promising target for the development of anti-aging medicines.

Reduced IIS confers lifespan extension in many species, potentially including humans (Fontana et al., 2010; Kenyon, 2010). In C. elegans, inhibition of daf-2, which encodes a homolog of insulin/lGF-1 receptor, doubles lifespan (Kenyon et al., 1993). This long lifespan requires the FOXO transcription factor DAF-16, which induces the expression of many pivotal target genes to promote longevity (Fontana et al., 2010; Kenyon, 2010). Heat shock factor 1 (HSF-1) is another transcription factor crucial for longevity downstream of IIS (Hsu et al., 2003; Morley \& Morimoto, 2004). HSF-1 is activated upon reduction in IIS and is required for the long lifespan caused by inhibition of IIS, including daf-2 mutations (Hsu et al., 2003; Morley \& Morimoto, 2004; Chiang et al., 2012). In addition, over-expression of hsf-1 prolongs the lifespan of C. elegans, suggesting that increase in HSF-1 activity is sufficient for longevity (Hsu et al., 2003; Morley \& Morimoto, 2004). It has been shown that HSF-1 not only promotes longevity but also enhances stress resistance, innate immunity, and protein homeostasis, and that these effects are achieved mainly through increasing the levels of molecular chaperones at the transcription level (Hsu et al., 2003; Cohen et al., 2006; Singh \& Aballay, 2006; Mohri-Shiomi \& Garsin, 2008; Steinkraus 
et al., 2008). These studies demonstrated that HSF-1 acts downstream of IIS to extend lifespan; however, whether other longevity signaling pathways, including the TOR pathway, also influence HSF-1 activity is poorly understood.

In this report, we show that HSF-1 mediates the lifespan extension caused by reduction of TOR signaling in C. elegans. We found that enhanced lifespan, oxidative stress resistance, and pathogen resistance resulting from inhibition of the TOR signaling pathway are highly dependent on HSF-1. In addition, we demonstrated that mutations in rsks-1, which encodes the C. elegans ribosomal protein 56 kinase, increase HSF-1 activity, and that hsp-16, a small heat-shock protein whose induction is regulated by HSF-1, is required for the longevity of rsks-1 mutants. Furthermore, we showed that HSF-1 is synergistically activated by the inhibition of both TOR signaling and IIS pathways and is required for the further enhanced longevity of daf-2 rsks-1 double mutants. Together, our findings establish HSF-1 as a hub protein for the cross talk between TOR signaling and IIS pathways to regulate lifespan.

\section{Results}

While performing a genome-wide RNA interference (RNAi) screen for identification of genes that interact with rsks-1, we found that RNAi targeting $h s f-1$ was one of the 12 RNAi clones that specifically reduced the body size of rsks-1 mutants (Fig. S1A). We confirmed this result using hsf-1(sy441) and rsks-1(tm1714) mutations (Fig. S1B). We were intrigued by this specific genetic interaction and further investigated the relationship between rsks-1 and hsf-1. Because C. elegans hsf-1 has been established as a crucial life-extending gene (Hsu et al., 2003; Morley \& Morimoto, 2004) and rsks-1 mutations prolong lifespan (Hansen et al., 2007; Pan et al., 2007), we examined the role of hsf-1 in the longevity of rsks-1 mutants. We found that hsf-1 RNAi or mutations completely suppressed the long lifespan of the rsks-1 mutants (Fig. 1AC). The effect of hsf-1 inhibition on the longevity of rsks-1 mutants was comparable to that of mutations in daf-16/FOXO transcription factor or aak-2/AMP-activated protein kinase (Fig. 1D,E and Fig. S2; see also Fig. S2 legend for discussion) (Selman et al., 2009). Knockdown of hsf-1 using RNAi only during adulthood completely suppressed the long lifespan of rsks-1 mutants as well (Fig. 1F and Fig. S1C). This result excludes the possibility that depletion of HSF-1 during larval development negatively affected the health of the rsks-1 mutant animals and subsequently reduced the lifespan during adulthood. Together, these data indicate that down-regulation of S6 kinase requires HSF-1 to lengthen lifespan in C. elegans.

Because S6K is a key downstream kinase of TORC1 (Laplante \& Sabatini, 2012), we asked whether hsf-1 plays a role in the longevity caused by down-regulation of other components in the TORC 1 signaling pathway. We found that $h s f-1$ was required for the lifespan extension caused by RNAi knockdown of daf-15/raptor or ragc-1/Rag GTPase, or by rapamycin treatment (Fig. $2 \mathrm{~A}-\mathrm{C}$ ). Down-regulation of TORC1 extends lifespan at least partly through decreasing mRNA translation, which is regulated by phosphorylation of ribosomal protein subunits such as RPS6 by S6K. We therefore examined the effects of hsf-1 mutations on the longevity caused by reduction in ribosomal protein subunit levels. We found that increased lifespan conferred by knockdown of rps-6 or rps-15 was suppressed by hsf-1 mutations (Fig. 2D,E). These results further support our notion that $h s f-1$ is crucial for lifespan extension by inhibition of TORC 1 signaling.

Longevity has been shown to correlate well with increased resistance against external stresses and protein aggregation. We therefore determined the role of $r s k s-1$ and hsf-1 in the survival rate under various stresses. Among the external stresses, rsks-1 mutations increased the resistance against oxidative stress but not against heat or osmotic stresses (Fig. 3A and Fig. S3A-C; see also Fig. S3 legend for discussion). We found that hsf-1 was required for the enhanced resistance of the rsks-1 mutants to oxidative stress (Fig. 3A). The requirement of hsf-1 for this increased oxidative stress resistance was comparable to or higher than that of daf-16/FOXO whose role in oxidative stress resistance is well known (Fig. 3B). We then examined the role of hsf-1 in enhanced protein homeostasis in rsks-1 mutants using polyglutamine-yellow fluorescent protein (Q40::YFP) transgenic animals (Morley et al., 2002), which are an established worm model of polyglutamine diseases. We found that rsks-1 mutations reduced Q40::YFP (Q40) aggregates and that $h s f-1$ was required for the reduction in this aggregate formation (Fig. 3C,D). These data suggest that rsks-1 mutations enhance oxidative stress resistance and protein homeostasis through HSF-1.

As innate immunity is influenced by various aging-regulatory genes including hsf-1, we also tested whether rsks-1 mutations affected the immunity against $P$. aeruginosa (PA14), a well-established model bacterial pathogen. We found that $r$ sks- 1 mutants were more resistant to PA14 than wild-type and that $h s f-1$ was absolutely required for this PA14 resistance (Fig. 4A). In contrast, RNAi targeting several other genes known to be crucial for pathogen resistance, such as daf-16/FOXO, nsy-1/MAP kinase kinase kinase, or pmk-1/MAP kinase (Irazoqui et al., 2010), did not fully suppress the PA14 resistance of rsks-1 mutants (Fig. 4B-D). Together, these data suggest that hsf-1 is essential for rsks-1 mutants to be resistant to oxidative stress, protein aggregation, and PA14. Given the strong correlation between longevity and stress resistance phenotypes in $C$. elegans, this enhanced resistance against various stresses may contribute to the long lifespan of rsks- 1 mutants.

The requirement of $h s f-1$ for the longevity, and stress and pathogen resistance caused by rsks-1 mutations led us to ask whether downregulation of TOR signaling was sufficient to increase HSF-1 activity. By performing real-time quantitative reverse transcription-PCR (qRT-PCR) analysis, we determined the mRNA expression levels of five known HSF-1 target chaperone genes, hsp-16.1/2/11 (hsp-16.1, hsp-16.2, and hsp16.11), hsp-70, hsp-12.6, aip-1, and sip-1 (Fig. 5A-E and Fig. S4). We found that $h s p-16.1 / 2 / 11$ and $h s p-70$ were significantly induced by rsks1 mutations in the presence and the absence of heat shock and that the induction was largely hsf-1 dependent (Fig. 5A,B and Fig. S4A,B). In contrast, $h s p-12.6$ and aip-1 were induced by rsks-1 mutations only upon heat-shock treatment (Fig. 5C,D and Fig. S4C,D). We also showed that GFP expression under the control of the $h s p-16.2$ promoter ( $h s p$ 16.2::GFP) (Link et al., 1999; Walker \& Lithgow, 2003), a fluorescence reporter for HSF-1 activity, was increased by rsks-1 mutations, and this up-regulation was suppressed by hsf-1 RNAi knockdown (Fig. 5F,G). Moreover, we found that $h s p-16.1 / 2 / 11$ and $h s p-70$ were induced by RNAi knockdown of daf-15 or ragc- 1 , and that hsf-1 mutations suppressed the induction of these genes (Fig. $5 \mathrm{H}-\mathrm{K}$ ). These data indicate that at least a subset of HSF-1 targets is induced by inhibition of TOR signaling.

Next, we examined the functional significance of the induction of $h s p-16$ and $h s p-70$ for the longevity of rsks-1 mutants. We found that RNAi targeting hsp-16.1/2/11 significantly suppressed the longevity of rsks-1 mutants but hsp-70 RNAi did not (Fig. 5L,M). Thus, rsks-1 mutants may live long, at least in part, through up-regulation of $h s p-16$ resulting from the activation of HSF-1.

HSF-1 is a well-known longevity transcription factor downstream of the IIS pathway (Hsu et al., 2003; Morley \& Morimoto, 2004; Chiang et al., 2012). We therefore explored the possibility that HSF-1 acts as a 
Fig. 1 HSF-1 is required for the longevity caused by the inhibition of $\mathrm{S} 6$ kinase (rsks1). (A, B) The long lifespan of $r s k s-1$ (tm1714) mutants was completely suppressed by hsf-1 RNAi (A) or hsf-1 (sy441) mutations (B). Because hsf-1(sy441) mutants display larval arrest phenotype at $25^{\circ} \mathrm{C}$ (Hajdu-Cronin et al., 2004), we tested whether the development of $h s f-1$ (sy441) mutants was affected at $22.5^{\circ} \mathrm{C}$. We found that $h s f-1$ (sy441) mutants did not display the larval arrest phenotype at $20{ }^{\circ} \mathrm{C}$ or $22.5^{\circ} \mathrm{C}$ (Fig. S1D), the

temperatures for the lifespan assays (A) and (B), respectively. (C) hsf-1 is required for the longevity of rsks-1 mutants without FUdR treatment. (D, E) The long lifespan of rsks-1 (tm1714) mutants was suppressed by daf16(mu86) (D) or aak-2(ok524) (E) mutations. (F) hsf-1 RNAi treatment only during adulthood (adult-only RNAi) suppressed the longevity of $r s k s-1(t m 1714)$ mutants. See also Fig. S1C for the lifespan results of rsks-1(tm1714) mutants treated with hsf-1 RNAi only during adulthood without FUdR. A summary of the lifespan data presented in this and other Figures and additional repeats are included in Table S1. Lifespan experiments were carried out at $20{ }^{\circ} \mathrm{C}$ for (A) and at $22.5^{\circ} \mathrm{C}$ for (B-F).
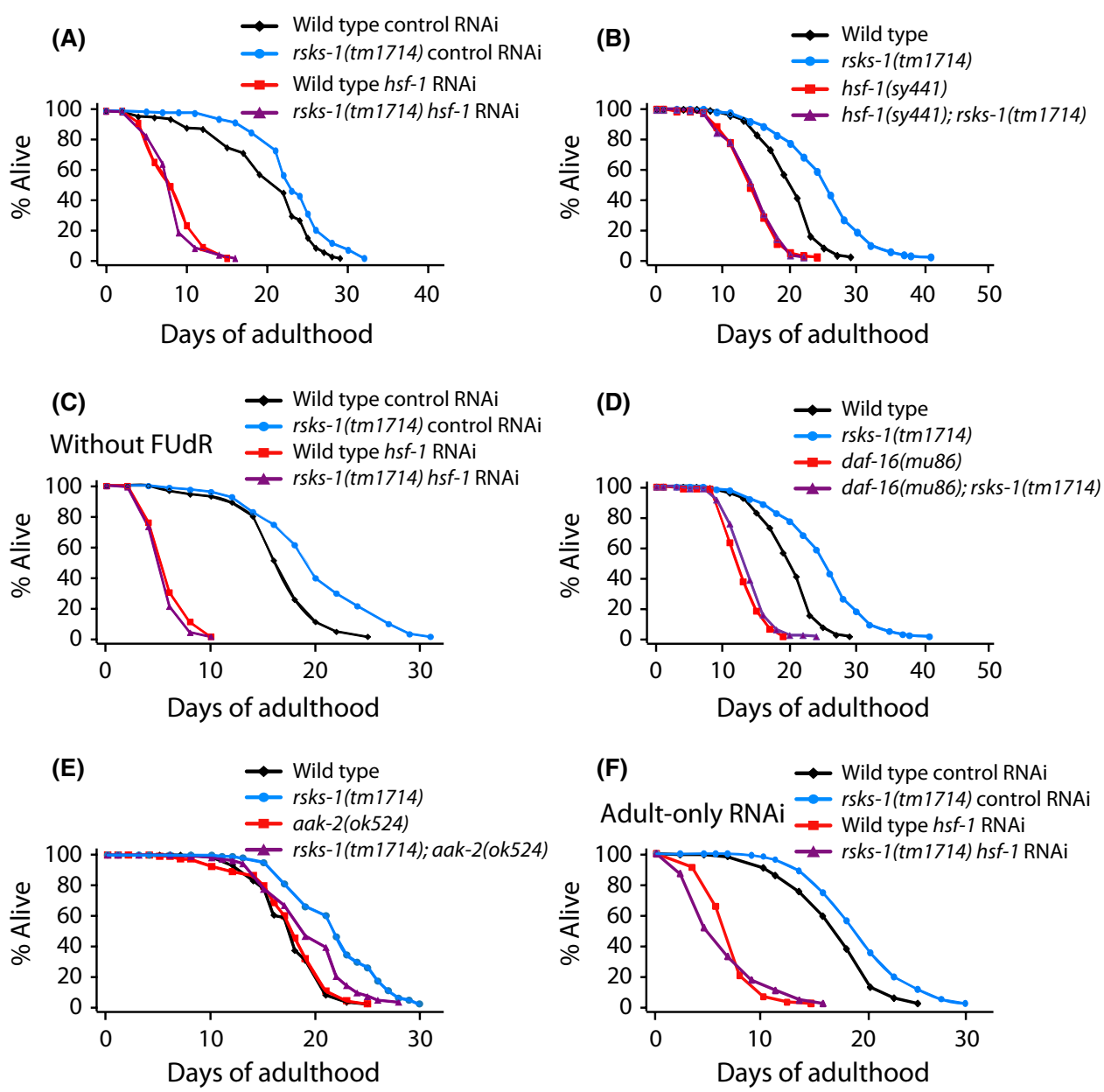

hub transcription factor for the cross talk between insulin/IGF-1 and TOR signaling pathways. Consistent with this idea, we found that the synergistic lifespan-extending effect by rsks-1 and daf-2 mutations was largely abolished by hsf-1 RNAi (Fig. 6A). In addition, daf-16 mutations fully suppressed the longevity of daf-2 rsks-1 double mutants (Fig. 6B). In contrast, enhanced dauer formation caused by rsks-1 mutations in daf-2 mutants, which requires daf-16, was not suppressed by hsf-1 RNAi (Fig. 6C). These data suggest that HSF-1 requirement for the enhanced longevity of the daf-2 rsks-1 double mutant is distinct compared with that of the dauer phenotype of the double mutant. We also found that $h s p-16.1 / 2 / 11$ and $h s p-70$ were further induced by rsks1 mutations in daf-2/insulin/IGF-1 receptor mutants and that these highly induced mRNA levels were largely suppressed by hsf-1 RNAi (Fig. 6D,E). Together, these data indicate that HSF-1 as well as DAF-16 acts as a key transcription factor mediating the synergistic longevity conferred by simultaneous down-regulation of both IIS and TOR pathways.

\section{Discussion}

In this study, we show that HSF-1 is a crucial longevity transcription factor acting downstream of the TOR signaling pathway. We found that genetic inhibition of $h s f-1$ completely abrogated the long lifespan caused by inhibition of TOR signaling components. We also demonstrated that inhibition of C. elegans S6 kinase (rsks-1), a key downstream kinase of TOR, increased the level of a subset of HSF-1 targets. In addition, hsf-1 was required for the resistance of rsks- 1 mutants to various internal and external stresses, which has been shown to strongly correlate with longevity. Furthermore, we showed that HSF-1 was required for the longevity caused by simultaneous inhibition of both IIS and TOR signaling. Together, our findings suggest that HSF-1 may act as a converging transcription factor downstream of these two most evolutionarily well-conserved longevity pathways (Fig. 6F).

We noted the specific genetic interaction between rsks-1 and hsf-1 initially through an unbiased modifier RNAi screen using the slowgrowth phenotype of $r s k s-1$ mutants. We then found the requirement of HSF-1 in other physiological phenotypes of rsks-1 mutants, including lifespan extension, stress resistance, and pathogen resistance. Because hsf-1 RNAi influenced the growth of rsks-1 mutants, HSF-1 might be considered a limiting factor for $r s k s-1$ mutants to normally develop to adulthood and to live long. However, we prefer our model proposing that HSF-1 acts as an instructive factor for the longevity and the stress resistance of rsks-1 mutants for the following reasons. First, we showed that hsf-1 RNAi only during adulthood was sufficient for suppressing the longevity of rsks-1 mutants, excluding the possibility that hsf-1 RNAi results in general sickness during growth. Second, we found that rsks-1 mutations increased a subset of HSF-1 target gene expression and that one HSF-1 target gene, $h s p-16$, was at least partly required for the long lifespan. Third, we showed that TOR signaling and IIS synergistically increased the level of HSF-1 target genes. These data are consistent with our model that the reduction in TOR signaling activates HSF-1, which in turn extends lifespan. 

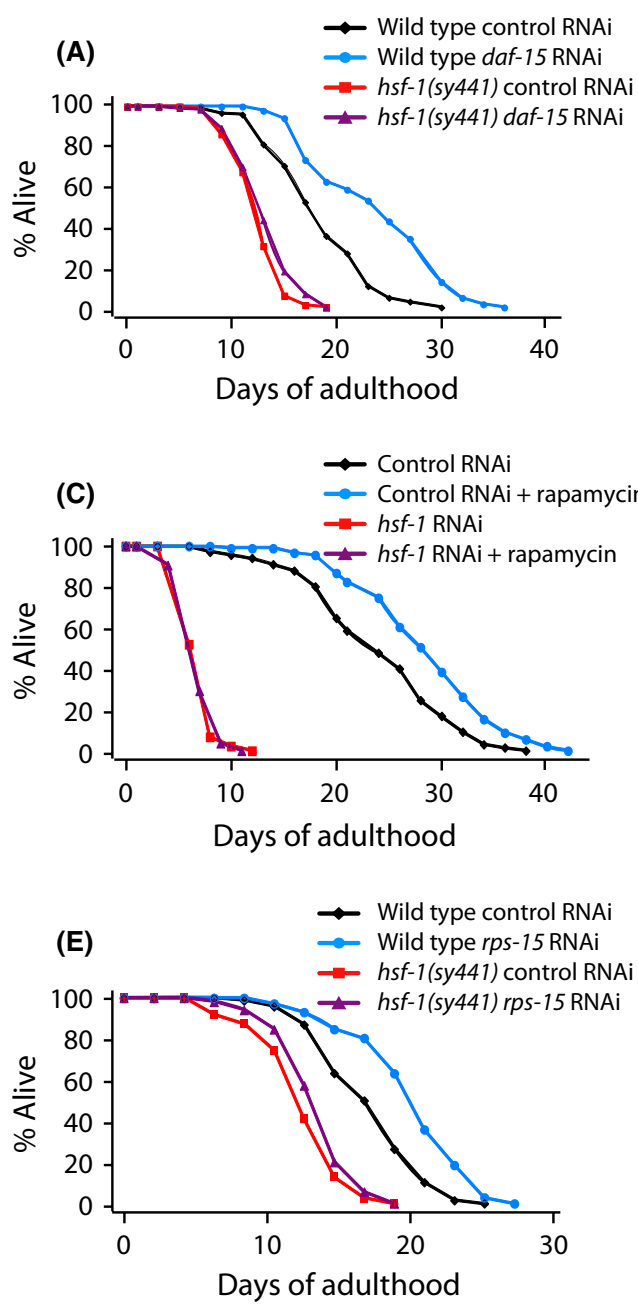

(A) Oxidative stress
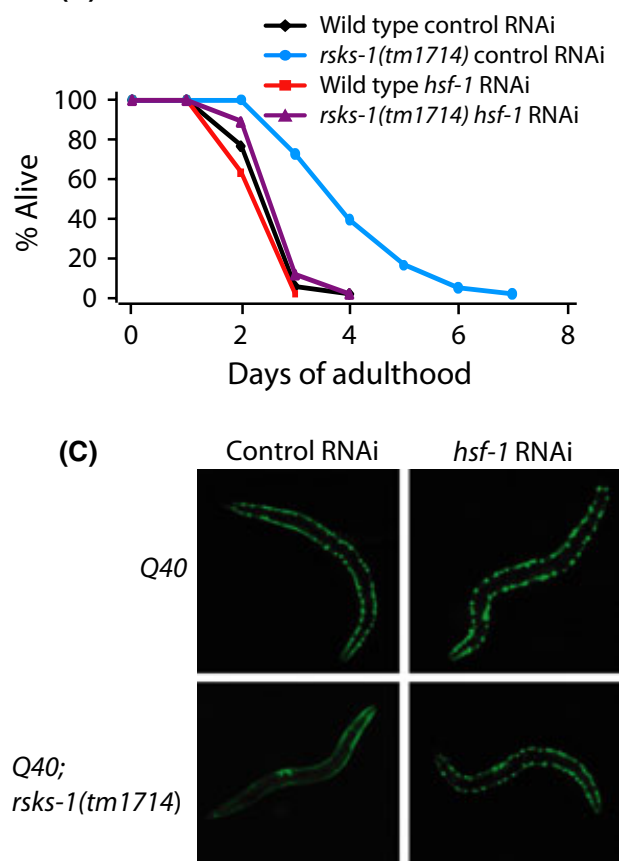
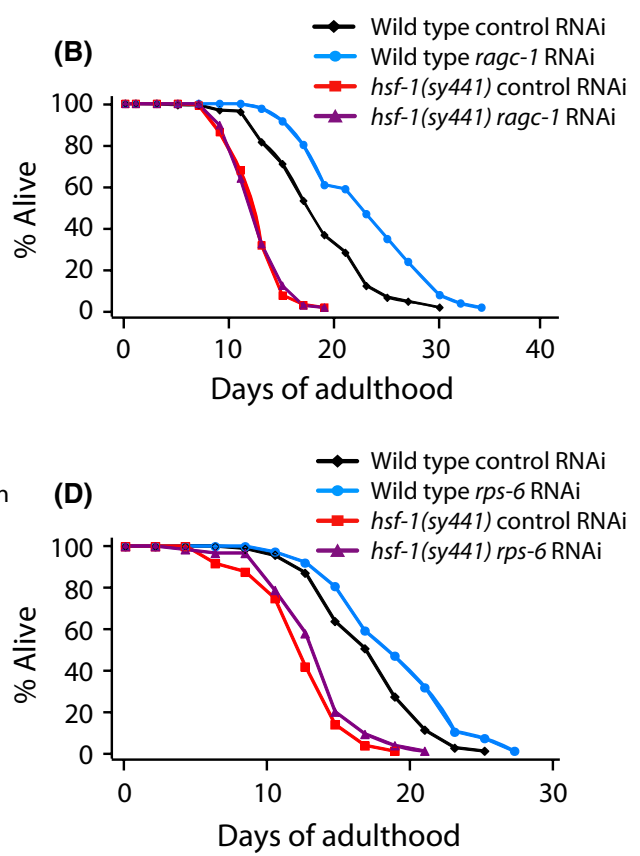

Fig. 2 HSF-1 is required for the long lifespan resulting from the inhibition of various components of TOR signaling. (A, B) Lifespan extension caused by inhibiting one of the components in TORC 1 required hsf-1. hsf-1(sy441) mutation suppressed the prolonged lifespan caused by RNAi targeting daf-15/raptor (A) or ragc-1/Rag GTPase (B). (C) The longevity conferred by treating wild type with rapamycin $(100 \mu \mathrm{m})$ was suppressed by RNAi knockdown of hsf1. (D, E) Long lifespan conferred by knockdown of rps-6 (D) or rps-15 (E) was dependent on hsf-1. Note that we carried out lifespan assays by treating animals with RNAi only during adulthood, because daf15 homozygous mutations or RNAi clones targeting ribosomal protein subunits result in larval arrest phenotypes (Jia et al., 2004, Hansen et al., 2007). All lifespan experiments in this figure were carried out at $22.5^{\circ} \mathrm{C}$.

\section{(B) Oxidative stress}

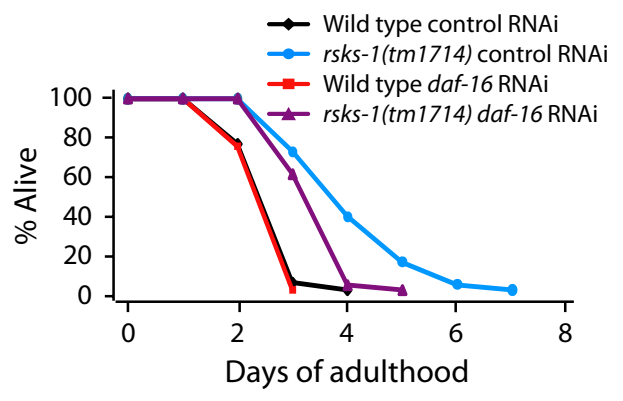

(D) $\square 40$

$\square$ Q40; rsks-1(tm1714)

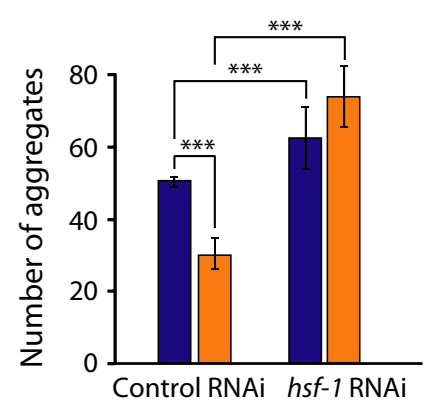

Fig. 3 Enhanced resistance of $r s k s-1$ mutants against oxidative stress and polyglutamine aggregation requires $h s f-1$. (A, B) Increased resistance of $r s k s-1$ (tm1714) mutants against oxidative stress caused by paraquat $(20 \mathrm{~mm}$ ) treatment was completely suppressed by hsf-1 RNAi (A) and partially suppressed by daf-16/FOXO RNAi (B). (C, D) rsks-1(tm 1714) mutation decreased the level of aggregated YFPfused polyglutamine repeats (Q40). (C) RNAi knockdown of $h s f-1$ reversed the reduced polyglutamine aggregation in rsks1(tm1714) mutant animals. (D)

Quantification of the number of Q40::YFP (Q40) aggregates. Error bars represent the standard error of the mean (s.e.m.) ( $n>30$, $* * * P<0.001$, two-tailed Student's $t$-test). Statistical analysis of the data presented in this figure and additional repeats are described in Table S2. 

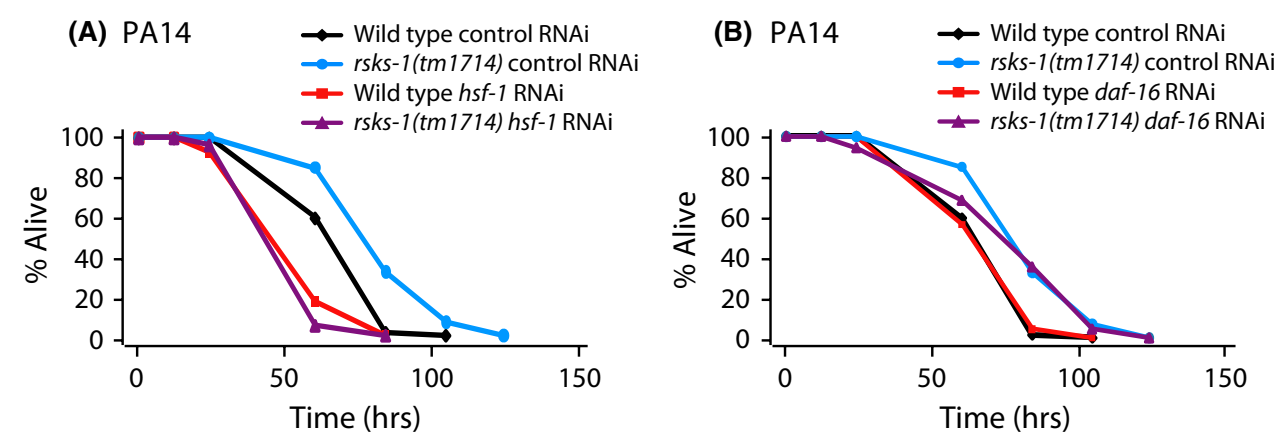

Fig. 4 Resistance to PA14 conferred by rsks-1 mutations requires $h s f-1$. (A, B) hsf-1 was required for the increased survival of rsks-1(tm1714) mutants feeding on the pathogen $P$. aeruginosa (PA14) (A), whereas daf-16/FOXO RNAi (B) had little effects on the survival. (C, D) RNAi targeting nsy-1/MAP kinase kinase kinase (C) or pmk-1/MAP kinase (D) did not fully suppress the pathogen resistance caused by rsks-1(tm1714) mutations. A summary of the data presented in this figure and additional repeats are described in Table S2.

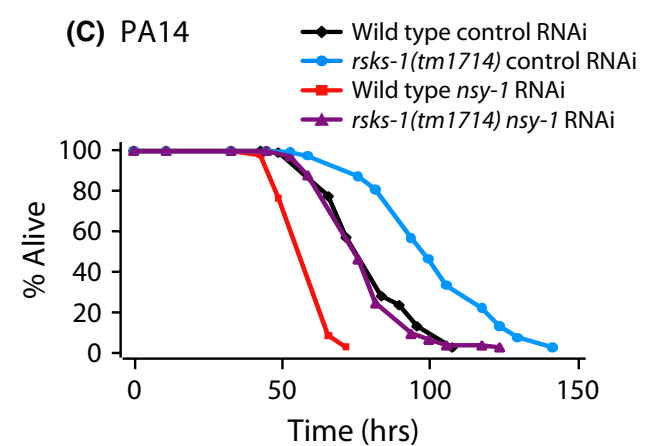

How does TOR signaling influence the activity of HSF-1? Although a recent study using cultured mammalian cells showed that mammalian TOR (mTOR) phosphorylates and activates HSF-1 (Chou et al., 2012), this may not be directly translated into our study. First, the serine residue in HSF-1 that is phosphorylated by mTOR (Chou et al., 2012) is not conserved across organisms, including yeast, C. elegans, Drosophila, and zebrafish. Thus, this regulatory mechanism of HSF-1 by TOR may have evolved relatively recently. Moreover, in contrast to Chou et al.'s paper suggesting the activation of HSF-1 by TOR, we found that reduction in TOR signaling activates HSF-1. Thus, the role of phosphorylation of HSF1 by TOR in lifespan regulation remains to be determined in future studies.

What will be the functional significance of up-regulation of HSF-1 activity by reduced TOR signaling? A previous report showed that dietary restriction increases lifespan and enhances resistance to polyglutamine aggregation in an hsf-1-dependent manner (Steinkraus et al., 2008). As dietary restriction reduces TOR signaling (Kaeberlein \& Kennedy, 2011), it seems likely that dietary restriction promotes longevity and stress resistance via inhibiting TOR signaling and subsequently increasing HSF-1 activity. Inhibition of TOR signaling is also well known to decrease mRNA translation, which leads to reduction in the overall protein level. Several studies have indicated that reduced translation by TOR signaling increases lifespan and stress resistance (Stanfel et al., 2009; Kapahi et al., 2010; Evans et al., 2011; Laplante \& Sabatini, 2012). Under these conditions, organisms may need to maintain biological functions using available proteins that were already synthesized. Therefore, the thrifty usage of the available proteins would be very critical for survival. Our data imply that reduced TOR signaling results in the activation of HSF-1 and subsequent up-regulation of molecular chaperones, which helps to improve overall protein quality control. This enhanced protein quality control in general may in turn increase lifespan and stress resistance. Alternatively, activated HSF-1 may induce specific chaperones, which facilitate maintaining or activating a subset of client proteins that are important for longevity and stress resistance. Consistent with this idea, only a subset of known HSF-1 target genes is induced by rsks-1 mutations. Moreover, a small heat-shock protein that has been shown to be necessary and sufficient for the longevity caused by reduced IIS (Hsu et al., 2003; Walker \& Lithgow, 2003; Morley \& Morimoto, 2004) was required for the long lifespan of $r s k s-1$ mutants. This indicates that the client proteins of HSP-16 are pivotal longevity proteins. It will be interesting to investigate these two alternative but not mutually exclusive possibilities to further understand the molecular basis of lifespan regulation by TOR signaling. Future studies using biochemical approaches to determine how the activity of HSF-1 is increased by reduced TOR signaling in animals will shed light on this issue.

Several previous studies have revealed that TOR signaling and IIS pathways employ common transcription factors, including SKN-1/NRF-2 and DAF-16/FOXO to regulate the lifespan of $C$. elegans (Sheaffer et al., 2008; Tullet et al., 2008; Wang et al., 2010; Robida-Stubbs et al., 2012). Here, we show that HSF-1 functions as an intersecting point or downstream of the intersecting point of TOR and IIS pathways. Future studies to elucidate the mechanisms by which these transcription factors interact with one another upon reduction in TOR signaling and IIS pathways will be important for providing insights into solving the secrets of human aging, particularly because TOR signaling and IIS pathways are two of the most crucial longevity pathways.

\section{Experimental procedures}

\section{Strains}

The following strains were analyzed in this study.

N2 wild-type, VB633 rsks-1(sv31) III, FX01714 rsks-1(tm1714) III, IJ109 rsks-1(tm1714) III outcrossed 4 times to Lee-lab N2, which is the same as Kenyon-lab N2, CF2725 aak-2(ok524) X outcrossed 3 times to Kenyon-lab N2, CF2495 hsf-1(sy441) I outcrossed three times to 
- Wild type
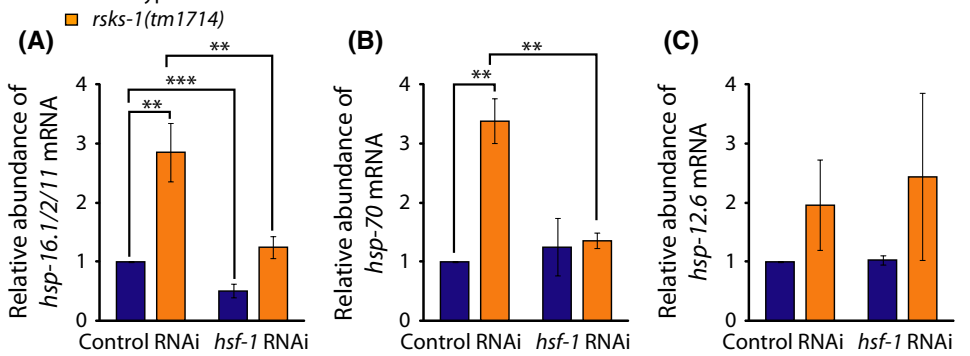

(D)

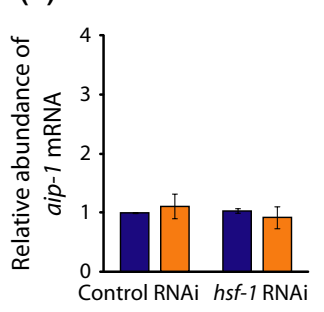

(E)

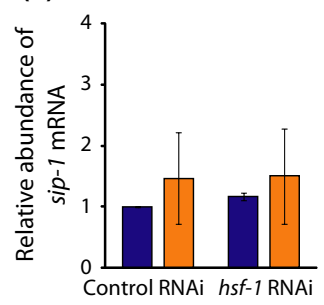

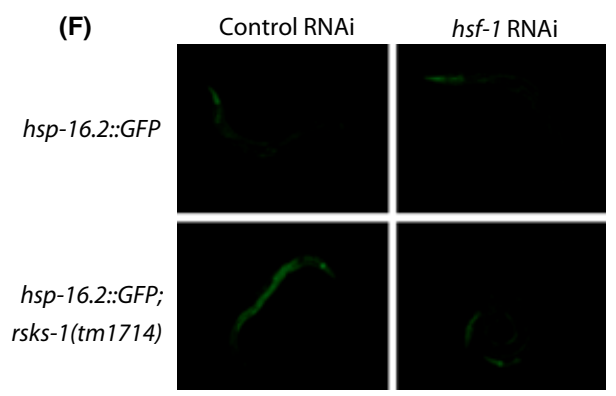

(G)

$\square h s p-16.2:: G F P$
$\square h s p-16.2:: G F P ; r s k s-1(t m 1714)$

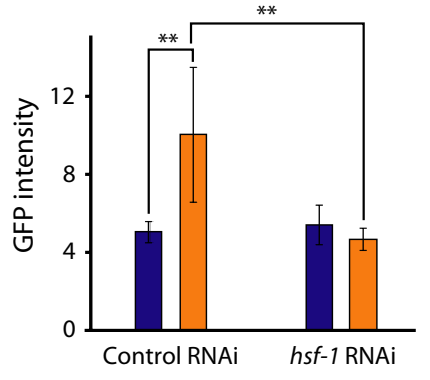

- Control RNAi

(J)

$\square$ ragc-1 RNAi

Control RNAi
adaf-15 RNAi

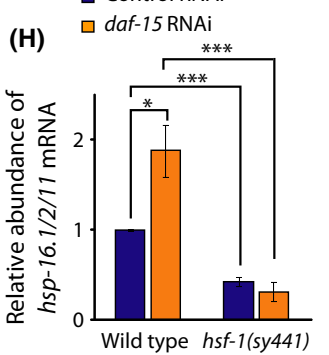

(I)

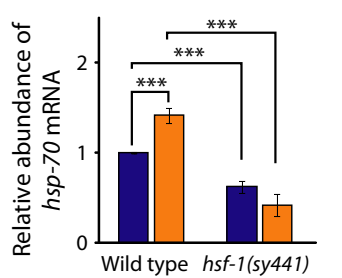

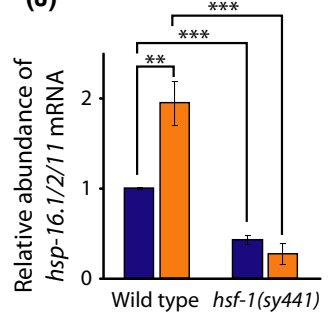

(K)

- Control RNAi

口 ragc-1 RNAi
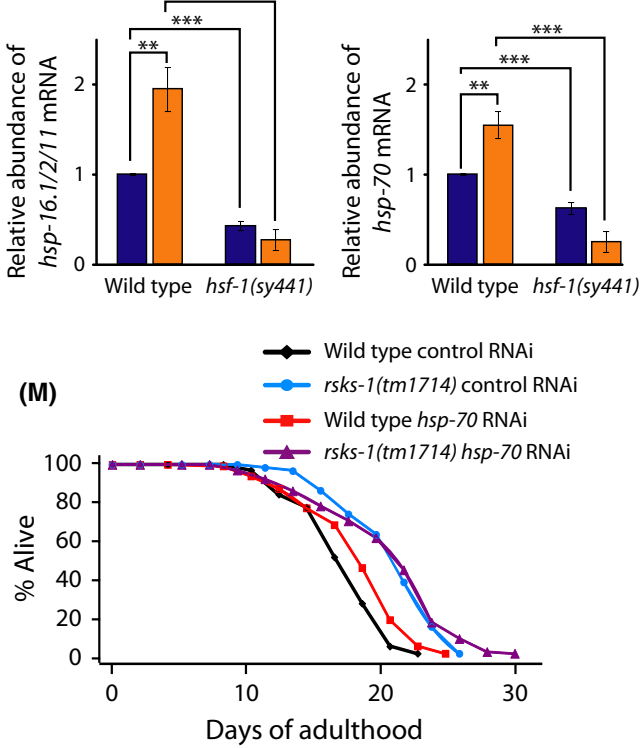

Fig. 5 rsks-1 mutations extend lifespan through increasing the level of small heat-shock proteins in an HSF-1-dependent manner. (A-E) Of five HSF-1 target genes that we tested, mRNA levels of $h s p-16.1 / 2 / 11$ (A) and $h s p-70$ (C12C8.1) (B) were highly increased by the rsks-1(tm 1714) mutations in an HSF-1-dependent manner in the absence of heat shock. mRNA expression of the other three tested HSF-1 target chaperon genes, hsp-12.6 (C), aip-1 (D), and sip-1 (E), was not significantly affected by rsks-1 mutations or hsf-1 RNAi. Error bars represent the standard error of the mean (s.e.m) $\left(n=4, * * P<0.01, * * * P<0.001\right.$, two-tailed Student's t-test). (F) After incubating animals for $1 \mathrm{~h}$ at $32{ }^{\circ} \mathrm{C}$, the level of GFP expression under the control of an hsp-16.2 promoter (hsp-16.2::GFP) was significantly increased by rsks-1 (tm 1714) mutations in an HSF-1-dependent manner. (G) Quantification of GFP pixel intensity. Experiments were performed three times independently, and error bars represent the standard error of the mean (s.e.m) ( $n>30$, $* * P<0.01$, two-tailed Student's t-test). (H, I) daf-15 RNAi increased the mRNA levels of hsp-16.1/2/11 (H) and hsp-70 (I) in an hsf-1-dependent manner. (J, K) Likewise, ragc-1 RNAi induced the mRNA expression of $h s p-16.1 / 2 / 11(\mathrm{~J})$ and $h s p-70(\mathrm{~K})$, and $h s f-1$ mutations suppressed the induction of these genes. Error bars represent the standard error of the mean (s.e.m) $(n=6, * p<0.05, * * p<0.01, * * * p<0.001$, two-tailed Student's t-test). (L) hsp-16.1/2/11 RNAi, which targets hsp-16.1, hsp-16.2, and hsp-16.11, suppressed the longevity conferred by rsks-1(tm1714) mutations in three of four trials (two of two trials at $20{ }^{\circ} \mathrm{C}$ and one of two trials at $22.5^{\circ} \mathrm{C}$.). We speculate that the

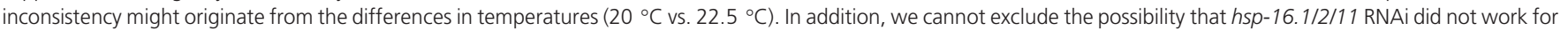

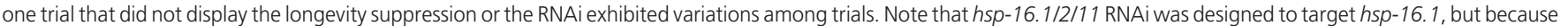
of over $88 \%$ sequence identity among $h s p-16.1$, hsp-16.2, and $h s p-16.11$ genes, the RNAi clone is expected to target $h s p-16.1$, $h s p-16.2$, and $h s p-16.11$ genes. (M) RNAi

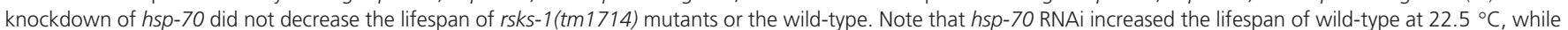

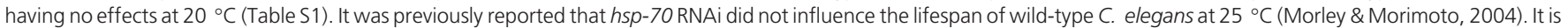
possible that $h s p$-70 RNAi may increase the lifespan of worms at an intermediate temperature $\left(22.5^{\circ} \mathrm{C}\right)$ via compensatory induction of life-extending genes, while not affecting lifespan at $20^{\circ} \mathrm{C}$ or $25^{\circ} \mathrm{C}$. Currently, we do not have mechanistic explanation for this result. All lifespan experiments in this figure were carried out at $22.5^{\circ} \mathrm{C}$. 
Fig. 6 HSF-1 is synergistically activated and is required for the extended longevity caused by simultaneous down-regulation of daf-2 and rsks-1. (A, B) rsks-1(tm1714) mutations further increased the long lifespan of daf-2(e1370) mutants. This very long lifespan was suppressed by hsf-1 RNA (A) or daf-16 mutations (B). (C) rsks-1 mutations enhanced dauer formation in daf-2 mutants. This increased dauer formation was not affected by hsf-1 RNAi in either daf-2(e1370) or daf-2(e1370) rsks1(tm1714) mutants. In contrast, daf-16 RNAi suppressed dauer formation in daf-2 (e1370) and daf-2(e1370) rsks-1(tm1714) mutants. Likewise, daf-16 mutations suppressed the dauer formation of rsks-1 single mutants at $25^{\circ} \mathrm{C}$ but $h s f-1$ RNAi did not (Fig. S5A,B). Error bars represent the standard error of the mean (s.e.m) $(n=4$, $* P<0.05, * * P<0.01, * * * P<0.001$, twotailed Student's t-test). (D, E) hsp-16.1/2/11 (D) and $h s p-70$ (E) mRNA levels were further increased by rsks-1(tm1714) mutations in daf-2(e1370) mutants, and hsf-1 RNAi nearly reversed the induction of these chaperone genes in daf-2(e1370) rsks-1(tm 1714) double mutants. Error bars represent the standard error of the mean (s.e.m) $(n=4, * P<0.05$, two-tailed Student's t-test). (F) Schematic

representation of the longevity pathways by which TOR signaling and IIS regulate HSF-1. Inhibition of DAF-2/insulin/IGF-1 receptor in the IIS or positive regulators of TOR signaling, including Rag GTPase, raptor, TOR kinase, and $\$ 6$ kinase, increases the activities of DAF-16 and HSF-1, which appears to lead to longevity. All lifespan experiments in this figure were carried out at $20^{\circ} \mathrm{C}$.
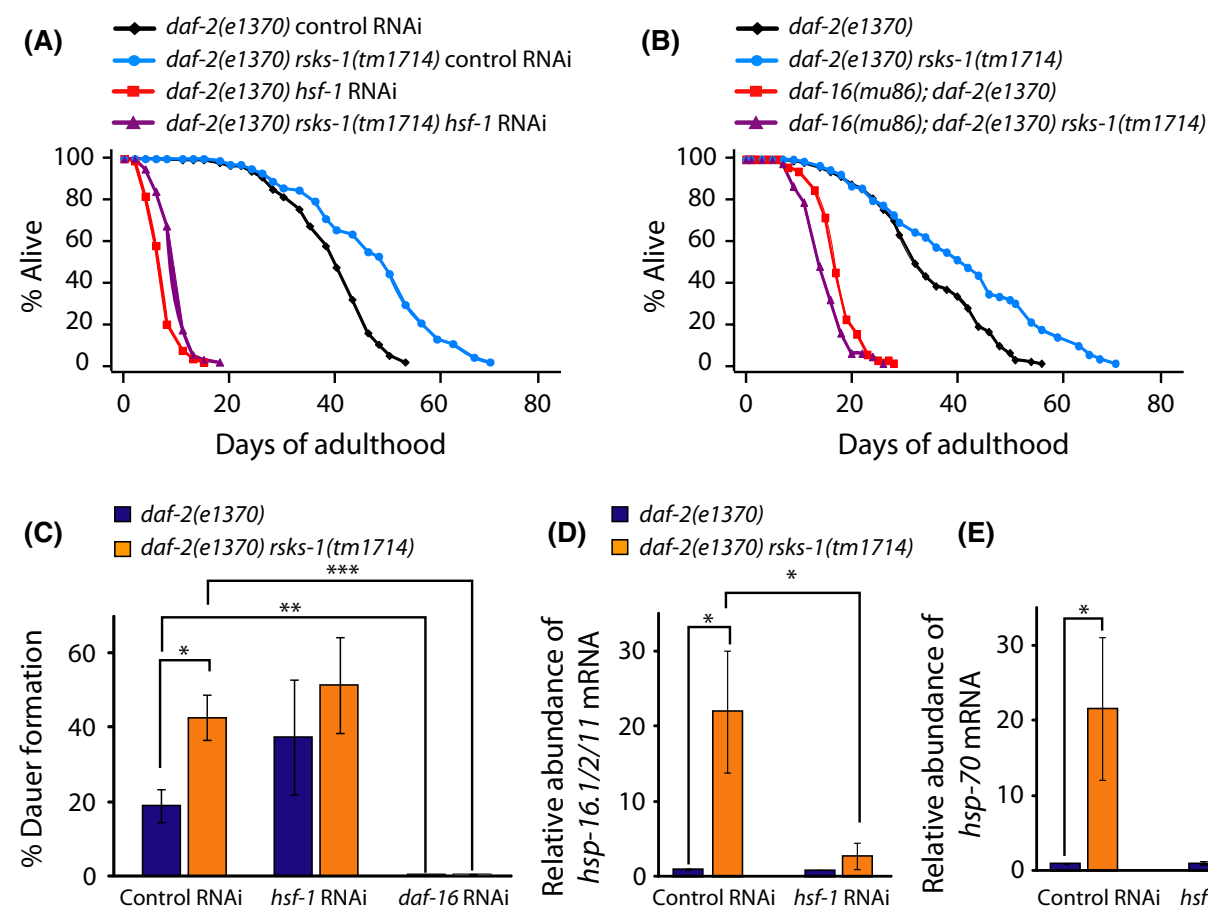

(D)

daf-2(e1370)

$\square$ daf-2(e1370) rsks-1(tm1714)

(E)
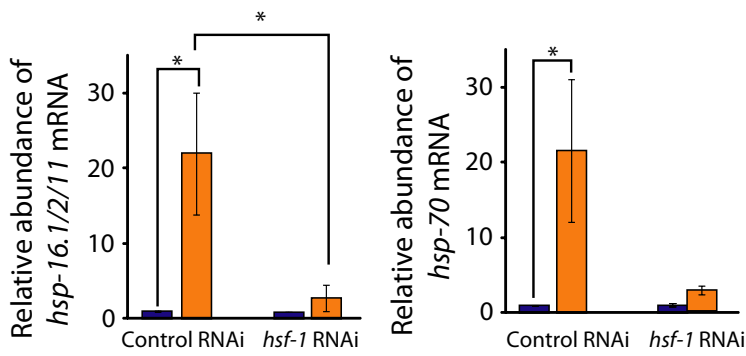

(F)

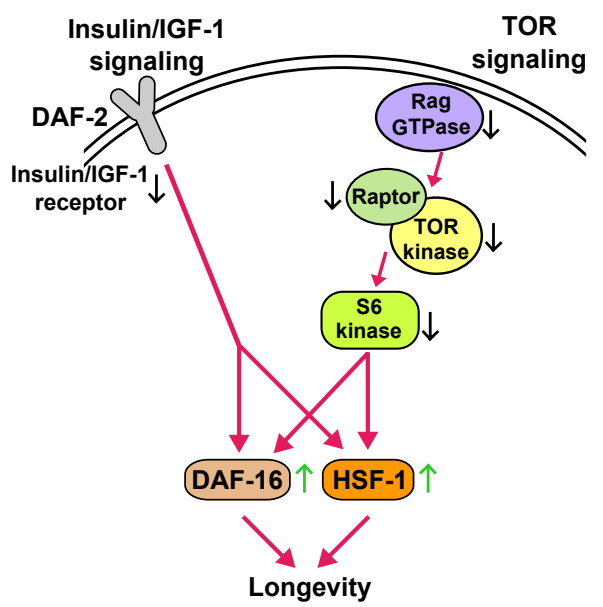

Kenyon-lab N2, CF1042 daf-16(mu86) I outcrossed 11 times to Kenyonlab N2, IJ184 rm/s133[unc-54p::Q40::YFP] outcrossed 4 times to Kenyon-lab N2, CL2070 dvls70[hsp-16.2p::GFP; rol-6D], IJ120 rsks-1 (tm1714) III; aak-2(ok524) X, IJ121 hsf-1(sy441) l; rsks-1(tm1714) III, IJ123 daf-16(mu86) I; rsks-1(tm1714) III, IJ185 rsks-1(tm1714) III; rmls133[unc-54p::Q40::YFP], IJ186 rsks-1(tm1714) III; dvls70[hsp16.2p::GFP; rol-6D], CF1041 daf-2(e1370) III outcrossed 6 times to Kenyon-lab N2, CF1085 daf-16(mu86) I; daf-2(e1370) III, IJ124 daf-2 (e1370) rsks-1(tm1714) III, IJ413 daf-16(mu86) I; daf-2(e1370) rsks-1 (tm1714) III.

\section{Lifespan assays}

Lifespan assays were performed as described previously with some modifications (Lee et al., 2010). Worms were synchronized by allowing gravid adults to lay eggs for $12 \mathrm{~h}$, and the progeny were allowed to develop. When worms were at young adult stage, approximately 30 worms were transferred onto each new NGM plate containing $5 \mu \mathrm{m} 2^{\prime}$ fluoro-5'deoxyuridine (FUdR, Sigma). For the experiment shown in Fig. $1 \mathrm{C}$ and Fig. S1C, approximately 30 worms were synchronized at young adult stage and worms were transferred to fresh plates without FUdR every $2-3$ days. We carried out lifespan assays at $20^{\circ} \mathrm{C}$ or $22.5^{\circ} \mathrm{C}$ for the following reasons. When we measured the lifespan of $r s k s-1$ mutants at $20^{\circ} \mathrm{C}$, the animals tend to display a vulval extrusion phenotype, which required censoring of many animals. To circumvent this problem, we performed the lifespan assays mostly at $22.5^{\circ} \mathrm{C}$ after maintaining the worms at $22.5^{\circ} \mathrm{C}$ for at least two generations. We carried out the lifespan experiments for daf-2(e1370) or daf-2(e1370) rsks-1(tm1714) mutants at $20^{\circ} \mathrm{C}$, because many of these animals arrested as dauer larvae at $22.5^{\circ} \mathrm{C}$. We specified the temperature at which each lifespan was measured in the Figure Legends and the Supporting Tables. Animals that ruptured, exhibited internal hatching of progeny, burrowed, or crawled off the plates were censored but included in the lifespan analysis as censored animals, as described 
previously (Lee et al., 2010). OASIS (online application of survival analysis, http://sbi.postech.ac.kr/oasis) was used for statistical analysis (Yang et al., 2011). P values were calculated using the log-rank (MantelCox method) test.

\section{Rapamycin treatment}

Rapamycin (LC Laboratories, Woburn, MA, USA) was dissolved in DMSO to obtain a $50 \mathrm{mg} \mathrm{mL}^{-1}$ stock solution and added onto plates to achieve the final concentration of $100 \mu \mathrm{m}$. The control plates were prepared to contain the same amount of DMSO without rapamycin.

\section{Quantitative RT-PCR assays}

Synchronized worms at young (day 1) adult stage were prepared for quantitative RT-PCR assays. RNA isoplus (Takara, Shiga, Japan) reagent was used for the extraction of total RNA from C. elegans. Total cDNA was synthesized using the reverse transcription system (Promega, Madison, WI, USA) and used as templates for qPCR with the SYBR green dye (Takara, Shiga, Japan). The PCR reaction was carried out in a StepOne Real-Time PCR system (Applied Biosystems, USA). mRNA of hsp-16.1/2/11, hsp-70, aip-1, sip-1, or hsp-12.6 was amplified with gene-specific primers and normalized to ama-1 mRNA level. The sequences of the gene-specific primers are described in the Supporting Information.

\section{Oxidative stress resistance assays}

Oxidative stress resistance assays were performed using paraquat as described previously with some modifications (Lee et al., 2010). Gravid worms were transferred onto NGM plates seeded with Escherichia coli to lay eggs for $12 \mathrm{~h}$. Progeny were grown on the plates to reach adulthood, and approximately 80 young (day 1) adults were transferred onto bacteria-containing plates treated with paraquat (methyl viologen; Sigma, St. Louis, MO, USA) at a final concentration of $20 \mathrm{~mm}$ and with $5 \mu \mathrm{m}$ FUdR. Worms were incubated at $20^{\circ} \mathrm{C}$ and scored as dead when they did not respond to prodding with a platinum pick. The survival assays were repeated at least twice. OASIS was used for statistical analysis, and $P$ values were calculated using the log-rank (Mantel-Cox method) test.

\section{PA14 slow-killing assays}

PA14 slow-killing assays were carried out as described previously with minor modifications (Tan et al., 1999). P. aeruginosa (PA14) was cultured in LB media at $37{ }^{\circ} \mathrm{C}$ overnight, and $5 \mu \mathrm{L}$ of the culture was seeded onto high-peptone $(0.35 \%)$ agar plates with $5 \mu \mathrm{m}$ FUdR. The bacteria were then incubated at $37{ }^{\circ} \mathrm{C}$ for $24 \mathrm{~h}$ followed by incubation at $25{ }^{\circ} \mathrm{C}$ for $8-16 \mathrm{~h}$ for slow-killing assay. Approximately 100 young adult worms were transferred onto two PA14-seeded plates for each experiment. Worms were scored alive if they responded to prodding. OASIS was used for statistical analysis, and $P$ values were calculated using the log-rank (Mantel-Cox method) test.

\section{Fluorescence microscopy analysis}

The number of polyglutamine (Q40::YFP) aggregates defined as discrete structures with edges recognizable from surrounding fluorescence was counted as described previously using Axio Scope A1 compound microscope (Zeiss, Oberkochen, Germany) (Hsu et al., 2003). For measuring the level of $h s p-16.2::$ GFP expression, young (day 1) adult worms were subjected to heat shock for $1 \mathrm{~h}$ at $32{ }^{\circ} \mathrm{C}$, then recovered at $20^{\circ} \mathrm{C}$ for $6 \mathrm{~h}$, and monitored for GFP level using the Axio Scope A1 compound microscope. The fluorescence pixel intensity was quantified using IMAGEJ software (Rasband, W.S., ImageJ, U. S. National Institutes of Health, Bethesda, Maryland, USA, http://rsb.info.nih.gov/ij/).

\section{Dauer assays}

Dauer assay was performed as described previously with some modifications (Gaglia et al., 2012). At least 150 worms were synchronized by allowing $15-30$ adult worms to lay eggs at $22.5^{\circ} \mathrm{C}$ for $6 \mathrm{~h}$. Because the rsks-1(tm1714) mutations caused developmental delay in daf-2 mutants, dauers were scored after 2 days for daf-2(e1370) mutants and 3 days for daf-2(e1370) rsks-1(tm1714) mutants. The assay was independently repeated four times.

\section{Acknowledgments}

This work was supported by the National Research Foundation of Korea (NRF) grant funded by the Korea government (MSIP) (NO. NRF2012R1A4A1028200), and by a grant of the Korean Health Technology R\&D Project, Ministry of Health \& Welfare, Republic of Korea (A111656) to S-J.L., and by the National Research Foundation of Korea (NRF) grant funded by the Korea government (MSIP) (NO. NRF-2010-0019706) to S.K.J. Some C. elegans strains used in this study were provided by Drs. Cynthia Kenyon and Shohei Mitani, and by the Caenorhabditis Genetics Center, which is funded by the NIH National Center for Research Resources. We thank Dr. Dennis Kim for providing PA14, and all Lee and Jang laboratory members for help and discussion.

\section{References}

Bjedov I, Toivonen JM, Kerr F, Slack C, Jacobson J, Foley A, Partridge L (2010) Mechanisms of life span extension by rapamycin in the fruit fly Drosophila melanogaster. Cell Metab. 11, 35-46.

Chen D, Pan KZ, Palter JE, Kapahi P (2007) Longevity determined by developmental arrest genes in Caenorhabditis elegans. Aging Cell 6, 525-533.

Chiang WC, Ching TT, Lee HC, Mousigian C, Hsu AL (2012) HSF-1 regulators DDL-1/2 link insulin-like signaling to heat-shock responses and modulation of longevity. Cell 148, 322-334.

Chou SD, Prince T, Gong J, Calderwood SK (2012) mTOR is essential for the proteotoxic stress response, HSF1 activation and heat shock protein synthesis. PLOS ONE 7, e39679.

Cohen E, Bieschke J, Perciavalle RM, Kelly JW, Dillin A (2006) Opposing activities protect against age-onset proteotoxicity. Science 313, 1604-1610.

Curran SP, Ruvkun G (2007) Lifespan regulation by evolutionarily conserved genes essential for viability. PLoS Genet. 3, e56.

Dazert E, Hall MN (2011) mTOR signaling in disease. Curr. Opin. Cell Biol. 23, 744-755

Erikson E, Maller JL (1985) A protein kinase from Xenopus eggs specific for ribosomal protein S6. Proc. Natl Acad. Sci. USA 82, 742-746.

Evans DS, Kapahi P, Hsueh WC, Kockel L (2011) TOR signaling never gets old: aging, longevity and TORC1 activity. Ageing Res. Rev. 10, 225-237.

Fabrizio P, Pozza F, Pletcher SD, Gendron CM, Longo VD (2001) Regulation of longevity and stress resistance by Sch9 in yeast. Science 292, 288-290.

Fontana L, Partridge L, Longo VD (2010) Extending healthy life span-from yeast to humans. Science $\mathbf{3 2 8}, 321-326$

Gaglia MM, Jeong DE, Ryu EA, Lee D, Kenyon C, Lee SJ (2012) Genes That Act Downstream of Sensory Neurons to Influence Longevity, Dauer Formation, and Pathogen Responses in Caenorhabditis elegans. PLoS Genet. 8, e1003133.

Hajdu-Cronin YM, Chen WJ, Sternberg PW (2004) The L-type cyclin CYL-1 and the heat-shock-factor HSF-1 are required for heat-shock-induced protein expression in Caenorhabditis elegans. Genetics 168, 1937-1949. 
Hansen M, Taubert S, Crawford D, Libina N, Lee SJ, Kenyon C (2007) Lifespan extension by conditions that inhibit translation in Caenorhabditis elegans. Aging Cell 6, 95-110.

Harrison DE, Strong R, Sharp ZD, Nelson JF, Astle CM, Flurkey K, Nadon NL, Wilkinson JE, Frenkel K, Carter CS, Pahor M, Javors MA, Fernandez E, Miller RA (2009) Rapamycin fed late in life extends lifespan in genetically heterogeneous mice. Nature 460, 392-395.

Hsu AL, Murphy CT, Kenyon C (2003) Regulation of aging and age-related disease by DAF-16 and heat-shock factor. Science 300, 1142-1145.

Irazoqui JE, Urbach JM, Ausubel FM (2010) Evolution of host innate defence: insights from Caenorhabditis elegans and primitive invertebrates. Nat. Rev. Immunol. 10, 47-58.

Jia K, Chen D, Riddle DL (2004) The TOR pathway interacts with the insulin signaling pathway to regulate $C$. elegans larval development, metabolism and life span. Development 131, 3897-3906.

Kaeberlein M, Kennedy BK (2011) Hot topics in aging research: protein translation and TOR signaling, 2010. Aging Cell 10, 185-190.

Kaeberlein M, Powers RW 3rd, Steffen KK, Westman EA, Hu D, Dang N, Kerr EO, Kirkland KT, Fields S, Kennedy BK (2005) Regulation of yeast replicative life span by TOR and Sch9 in response to nutrients. Science 310, 1193-1196.

Kapahi P, Zid BM, Harper T, Koslover D, Sapin V, Benzer S (2004) Regulation of lifespan in Drosophila by modulation of genes in the TOR signaling pathway. Curr. Biol. 14, 885-890.

Kapahi P, Chen D, Rogers AN, Katewa SD, Li PW, Thomas EL, Kockel L (2010) With TOR, less is more: a key role for the conserved nutrient-sensing TOR pathway in aging. Cell Metab. 11, 453-465.

Kenyon CJ (2010) The genetics of ageing. Nature 464, 504-512.

Kenyon C, Chang J, Gensch E, Rudner A, Tabtiang R (1993) A C. elegans mutant that lives twice as long as wild type. Nature 366, 461-464.

Kim E, Goraksha-Hicks P, Li L, Neufeld TP, Guan KL (2008) Regulation of TORC 1 by Rag GTPases in nutrient response. Nat. Cell Biol. 10, 935-945.

Laplante M, Sabatini DM (2012) mTOR signaling in growth control and disease. Cell 149, 274-293.

Lee SJ, Hwang AB, Kenyon C (2010) Inhibition of respiration extends C. elegans life span via reactive oxygen species that increase HIF-1 activity. Curr. Biol. 20, 2131-2136.

Link CD, Cypser JR, Johnson CJ, Johnson TE (1999) Direct observation of stress response in Caenorhabditis elegans using a reporter transgene. Cell Stress Chaperones 4, 235-242.

Mohri-Shiomi A, Garsin DA (2008) Insulin signaling and the heat shock response modulate protein homeostasis in the Caenorhabditis elegans intestine during infection. J. Biol. Chem. 283, 194-201.

Morley JF, Morimoto RI (2004) Regulation of longevity in Caenorhabditis elegans by heat shock factor and molecular chaperones. Mol. Biol. Cell 15 657-664.

Morley JF, Brignull HR, Weyers JJ, Morimoto RI (2002) The threshold for polyglutamine-expansion protein aggregation and cellular toxicity is dynamic and influenced by aging in Caenorhabditis elegans. Proc. Natl Acad. Sci. USA 99, 10417-10422.

Pan KZ, Palter JE, Rogers AN, Olsen A, Chen D, Lithgow GJ, Kapahi P (2007) Inhibition of mRNA translation extends lifespan in Caenorhabditis elegans. Aging Cell 6, 111-119.

Powers RW 3rd, Kaeberlein M, Caldwell SD, Kennedy BK, Fields S (2006) Extension of chronological life span in yeast by decreased TOR pathway signaling. Genes Dev. 20, 174-184.

Robida-Stubbs S, Glover-Cutter K, Lamming DW, Mizunuma M, Narasimhan SD, Neumann-Haefelin E, Sabatini DM, Blackwell TK (2012) TOR signaling and rapamycin influence longevity by regulating SKN-1/Nrf and DAF-16/FoxO. Cell Metab. 15, 713-724.
Selman C, Tullet JM, Wieser D, Irvine E, Lingard SJ, Choudhury Al, Claret M, Al-Qassab H, Carmignac D, Ramadani F, Woods A, Robinson IC, Schuster E, Batterham RL, Kozma SC, Thomas G, Carling D, Okkenhaug K, Thornton JM, Partridge L, Gems D, Withers DJ (2009) Ribosomal protein S6 kinase 1 signaling regulates mammalian life span. Science 326, 140-144.

Sheaffer KL, Updike DL, Mango SE (2008) The Target of Rapamycin pathway antagonizes pha-4/FoxA to control development and aging. Curr. Biol. 18, 1355-1364.

Singh V, Aballay A (2006) Heat-shock transcription factor (HSF)-1 pathway required for Caenorhabditis elegans immunity. Proc. Natl Acad. Sci. USA 103, $13092-13097$.

Stanfel MN, Shamieh LS, Kaeberlein M, Kennedy BK (2009) The TOR pathway comes of age. Biochim. Biophys. Acta 1790, 1067-1074.

Steinkraus KA, Smith ED, Davis C, Carr D, Pendergrass WR, Sutphin GL, Kennedy BK, Kaeberlein M (2008) Dietary restriction suppresses proteotoxicity and enhances longevity by an hsf-1-dependent mechanism in Caenorhabditis elegans. Aging Cell 7, 394-404.

Syntichaki P, Troulinaki K, Tavernarakis N (2007) elF4E function in somatic cells modulates ageing in Caenorhabditis elegans. Nature 445, 922-926.

Tan MW, Rahme LG, Sternberg JA, Tompkins RG, Ausubel FM (1999) Pseudomonas aeruginosa killing of Caenorhabditis elegans used to identify $P$. aeruginosa virulence factors. Proc. Natl Acad. Sci. USA 96, 2408-2413.

Tullet JM, Hertweck M, An JH, Baker J, Hwang JY, Liu S, Oliveira RP, Baumeister R, Blackwell TK (2008) Direct inhibition of the longevity-promoting factor SKN-1 by insulin-like signaling in C. elegans. Cell 132, 1025-1038.

Walker GA, Lithgow GJ (2003) Lifespan extension in C. elegans by a molecular chaperone dependent upon insulin-like signals. Aging Cell 2, 131-139.

Wang J, Robida-Stubbs S, Tullet JM, Rual JF, Vidal M, Blackwell TK (2010) RNAi screening implicates a SKN-1-dependent transcriptional response in stress resistance and longevity deriving from translation inhibition. PLoS Genet. 6, e1001048.

Yang JS, Nam HJ, Seo M, Han SK, Choi Y, Nam HG, Lee SJ, Kim S (2011) OASIS: online application for the survival analysis of lifespan assays performed in aging research. PLOS ONE 6, e23525.

\section{Supporting Information}

Additional Supporting Information may be found in the online version of this article at the publisher's web-site.

Fig. S1 Genetic interaction between hsf-1 and rsks-1 for growth during development and adult lifespan phenotypes.

Fig. S2 Decrease in the lifespan of rsks-1 mutants by daf-16 RNAi treatment.

Fig. S3 The effects of rsks-1 mutations on the survival responses to heat and osmotic stresses.

Fig. S4 rsks-1 mutations induce a subset of HSF-1 target genes in an hsf-1dependent manner upon treatment with heat shock.

Fig. S5 Increased dauer formation by rsks-1 mutation requires daf-16 but not hsf-1.

Table S1 Lifespan analysis.

Table S2 Survival analysis other than lifespan analysis.

Data S1 Experimental procedures. 